\title{
Structural Characteristics Analysis of Hydraulic Lifting System of a Forklift
}

\author{
Wang Yao ${ }^{1}$, Zhang Qijiu' ${ }^{1}$ Xu Miao², Gan Xinji ${ }^{1}$, Wang Licai ${ }^{1}$, Bai Yan ${ }^{3, *}$ \\ ${ }^{1}$ School of Mechanical Engineering, Beihua University, Jilin, China \\ ${ }^{2}$ School of automotive and architectural engineering, Beihua University, Jilin, China \\ ${ }^{3}$ Engineering Training Center, Beihua University, Jilin, China \\ *Corresponding author
}

Keywords: Forklift, structural characteristics, controllability, observability

\begin{abstract}
In this paper, the structural characteristics of lifting mechanism of LiuGong CPD30 forklift are analyzed. After detailed analysis and calculation, it can satisfy the controllability and observability discriminant matrix. The structural characteristics of the hydraulic lifting system of forklift truck are analyzed and studied, which lays a theoretical foundation for the optimal design of the lifting mechanism of the forklift truck.
\end{abstract}

\section{Introduction}

The structural characteristics analysis of forklift truck hydraulic lifting system can make the analysis and synthesis of the system will be established on a strict theoretical basis. Controllability and observability are two important concepts in modern control theory. In modern control theory, the control of the state, that is whether the state vector can be controlled by the controlled quantity, and whether the state vector can be observed by the output, is entirely determined by the structural characteristics of the controlled system. Lifting the goods to a certain height or moving the goods from high to low is the most common working condition of forklift [1-3]. It requires forklift lifting system. However, due to the different weight of lifting goods and different lift heights, study the structural characteristics of forklift lifting system is necessary. And the controllability and observability of the system is verified by numerical calculation. It lays a solid theoretical foundation for solving the pole assignment and optimal control of the system in the future [4][5].

\section{Hydraulic System Structure Parameters}

Table 1 Lifting hydraulic cylinder parameters

\begin{tabular}{ll}
\hline Lifting hydraulic cylinder parameters & value \\
\hline Number & 2 \\
Maximum output force requirement & $32400 \mathrm{~N}$ \\
Maximum speed requirement & $0.15 \mathrm{~m} / \mathrm{s}$ \\
Maximum displacement requirement & $1.5 \mathrm{~m}$ \\
Piston diameter & $50 \mathrm{~mm}$ \\
Piston rod diameter & $25 \mathrm{~mm}$ \\
Rated pressure & $25 \mathrm{MPa}$ \\
System back pressure & $11 \mathrm{MPa}$ \\
Effective working length & $1600 \mathrm{~mm}$ \\
flow & $294.375 \mathrm{ml} / \mathrm{s}$ \\
\hline
\end{tabular}

The motor output torque on the oil pump, oil pump rod chamber for the hydraulic system oil flows through the valve into the hydraulic cylinder. And the oil cavity of the piston rod by squeezing through the reversing valve back into the tank. The motor torque to drive the pump pressure and the oil return tank pressure into the piston rod thrust acting on the movable pulley, the pulley thrust effect reduced to fork lift is accomplished. Based on the part of the lifting system of lift and lifting power up, according to the load of the hydraulic cylinder hydraulic cylinder to 
determine the rated pressure for the hydraulic pump, according to the determined cylinder pressure and flow of hydraulic system and the requirements for determining the rated pressure, displacement. Finally, the main parameters of the hydraulic cylinder and the oil pump are listed in Table 1 and Table 2.

Table 2 Hydraulic pump parameters

\begin{tabular}{ll}
\hline Hydraulic pump parameters & value \\
\hline Number & 1 \\
Flow requirement & $588.75 \mathrm{ml} / \mathrm{s}$ \\
Pressure requirement & $25 \mathrm{MPa}$ \\
model number & $\mathrm{CB}-\mathrm{FD} 16$ \\
displacement & $16 \mathrm{ml} / \mathrm{r}$ \\
rev & $600 \sim 3000 \mathrm{rpm}$ \\
Volume efficiency & $>90 \%$ \\
Total efficiency & $>81 \%$ \\
Actual flow & $144 \sim 720 \mathrm{ml} / \mathrm{s}$ \\
Actual pressure & $14.4 \sim 22.56 \mathrm{MPa}$ \\
\hline
\end{tabular}

\section{Controllability Analysis of Hydraulic Lifting System}

Controllability refers to the possibility that the control function controls the state of the controlled system. The complete controllability of the lifting system means that for any initial state of the system at any initial time, an admissible quantity of control can always be found, so that it can reach the target state in a limited time.

For the lifting system, the system matrix A and the control matrix B are under an initial state X. If in the time domain $\left[\mathrm{t}_{0}, \mathrm{t}_{\mathrm{f}}\right]$, the $\mathrm{X}(\mathrm{t})$ is transferred to $\mathrm{X}\left(\mathrm{t}_{\mathrm{f}}\right)=0$ through the control function $\mathrm{u}$, and the system is controllable. The lifting system can be described by the standard equations of controllability:

$$
\left\{\begin{array}{l}
\dot{X}=A X+B U \\
Y=M X+N U
\end{array}\right.
$$

In the upper form, the state vector $X$ :

$$
X=\left[\begin{array}{lll}
q_{7} & q_{13} & p_{23}
\end{array}\right]^{T}
$$

Control vector $u$ :

$$
u=\left[\begin{array}{llll}
S e_{1} & S e_{2} & S e_{3} & S e_{4}
\end{array}\right]^{T}
$$

System matrix A:

$$
A=\left[\begin{array}{ccc}
-\frac{1}{C_{7} \cdot R_{5}} & 0 & -\frac{1}{i_{4} \bullet i_{2} \bullet i_{23}} \\
0 & -\frac{1}{C_{13} \bullet R_{11}} & -\frac{1}{i_{4} \bullet i_{3} \bullet i_{23}} \\
\frac{i_{4} \bullet I_{23}}{\left(i_{4}{ }^{2} \cdot I_{23}+I_{17}\right) C_{7} \bullet i_{2}} & \frac{i_{4} \bullet I_{23}}{\left(i_{4}^{2} \bullet I_{23}+I_{17}\right) C_{13} \bullet i_{3}} & \frac{\left(i_{4}-1\right)^{2} \cdot R_{22}}{\left(i_{4}^{2} \cdot I_{23}+I_{17}\right) C_{7} \bullet i_{2}}
\end{array}\right]
$$

Control matrix B: 


$$
B=\left[\begin{array}{cccc}
\frac{1}{i_{1} \cdot R_{5}} & 0 & 0 & 0 \\
0 & \frac{1}{R_{11}} & 0 & 0 \\
0 & 0 & -\frac{1}{i_{4}} & 1
\end{array}\right]
$$

Output vector y:

$$
Y=f_{23}
$$

Output matrix M:

$$
M=\left[\frac{1}{I_{23}}\right]
$$

Direct transfer matrix N:

$$
N=[0]
$$

when the lifting height is 3 meters and the load is 3 tons, the matrix A and B are calculated:

$$
\begin{array}{r}
A=\left[\begin{array}{ccc}
-17857.14286 & 0 & -3.27249235 \times 10^{-7} \\
0 & -17857.14286 & -0.11317685 \\
674753112021.2 & 2.3335862 \times 10^{17} & 1124588.52004
\end{array}\right] \\
B=\left[\begin{array}{cccc}
1.0017834 \times 10^{-5} & 0 & 0 & 0 \\
0 & 2.55102041 \times 10^{-11} & 0 & 0 \\
0 & 0 & -0.5 & 1
\end{array}\right]
\end{array}
$$

According to the controllability criterion, the controllability discriminant matrix Qc is calculated:

$Q_{c}=\left[\begin{array}{lllll}B & A B & A^{2} B & \cdots \cdots & A^{n-1} B\end{array}\right]$

$=\left[\begin{array}{ccccccccccccc}1.0018 \times 10^{-5} & 0 & 0 & 0 & -0.1789 & 1.6362 \times 10^{-7} & 0 & -3.2725 \times 10^{-7} & 3192.25 & -1.767 & 0 & -0.362177 \\ 0 & 2.551 \times 10^{-11} & 0 & 0 & 0 & 0.056588 & 0 & -0.113177 & -765026.2 & -611116.54 & 0 & -125256.37 \\ 0 & 0 & -0.5 & 1 & 6759564.5 & 0 & 5390731.8 & 1124588.5 & 7.481 \times 10^{12} & 0 & 1.3211 \times 10^{16} & -2.641 \times 10^{16}\end{array}\right]$

It is obvious that the discriminant matrix is full rank $\left(R_{Q c}=3\right)$, so that the electric drive lifting system is completely controllable.

\section{Observe Ability Analysis of Hydraulic Lifting System}

Observability is the possibility that a system measure determines the system state.. The complete observability of the system is that the system can only be determined by the input and output in a limited time in any initial state of the system.

For the lifting systems, the system matrix $\mathrm{A}$, the output matrix $\mathrm{M}$, and an initial state $\mathrm{x}$ can be determined by the output y measured in the time domain $\left[t_{0}, t_{f}\right]$, the system is observable. The observe ability discriminant matrix $Q_{o}$ is calculated according to observability criterion: 


$$
Q o=\left[\begin{array}{c}
M \\
M A \\
M A^{2} \\
\vdots \\
M A^{n-1}
\end{array}\right]=\left[\begin{array}{ccc}
2 & 0 & 0 \\
-35714.28572 & 0 & -0.00000065 \\
637313477.365 & -152732859751.3114 & -0.724354
\end{array}\right]
$$

It is obvious that the discriminant matrix is full $\operatorname{rank}\left(R_{Q_{o}}=3\right)$, so it is known that the electric drive lifting system is observable.

\section{Conclusion}

In this paper, the structural characteristics of the forklift lift system are analyzed in detail. The numerical calculation shows that the system has complete controllability and observability.

\section{Acknowledgment}

The research is funded by the project development plan of science and technology in Jilin Province (20160418024FG).

\section{References}

[1] Wang Y, Zhao D, Wang L, et al. Dynamic simulation and analysis of the elevating mechanism of a forklift based on a power bond graph[J]. Journal of Mechanical Science \& Technology, 2016, 30(9):4043-4048.

[2] Du Guiping, Zhang Bo, Zhang Yongping. Matrix coefficient polynomial description model of DC-DC converters based on switched linear systems[J]. Proceedings of the CSEE, 2006, 26(21): 65-70

[3] Si J, Jiang W. CONTROLLABILITY AND OBSERVABILITY FOR A CLASS OF FRACTIONAL-ORDER IMPULSIVE SYSTEMS [J]. Annals of applied mathematics, 2012, 1(1): 77-86.

[4] Guan Z H, Qian T H, Yu X. On controllability and observability for a class of impulsive systems [J]. Systems \& Control Letters, 2002, 47(3):247-257.

[5] Klamka J, Babiarz A, Niezabitowski M. Banach fixed-point theorem in semilinear control ability problems-a survey [J]. Bulletin of the Polish Academy of Sciences Technical Sciences, 2016, 64(1):21-35. 